DOI:

10.1038/nrg2115

\section{URLs}

KaiA

http://www.expasy.org/

uniprot/Q2JTE8

KaiB

http://www.expasy.org/

uniprot/Q2JTE9

KaiC

http://www.expasy.org/

uniprot/Q2JTF0

\title{
Setting the pace: three's company
}

Circadian clocks regulate the daily rhythm of many biological processes. Timing is set through biochemical oscillators that sense and adjust to different environmental cues. A recent study now defines the molecular mechanisms of an oscillator that can comprise just three clock proteins and be reconstituted in a test tube.

Previous studies showed that cyanobacterial clock proteins KaiA, $\mathrm{KaiB}$ and KaiC are encoded by three clustered genes that are essential for the clock function. KaiC is a doubledoughnut-shaped homohexamer that undergoes rhythmical oscillations between phosphorylated states, which correspond to the period of the clock. KaiA and KaiB modulate KaiC phosphorylation by increasing or decreasing it, respectively. Interestingly, KaiC autophosphorylates and autodephosphorylates in vitro, making it possible to study in a test tube the rhythmic association patterns between the three Kai proteins according to the different phosphorylation states of KaiC.

Mori et al. used electron microscopy to track KaiABC complexes during an in vitro circadian cycle and found that various macromolecular assemblies were simultaneously present at each stage of KaiC phosphorylation. These data, and a further electrophoresis-based quantitative analysis of such complexes, allowed the authors to characterize the molecular components of the complexes at each stage. From this, they proposed a model to explain the dynamics of the in vitro KaiABC oscillator using a stochastic simulation that keeps track of the phosphorylation status of individual KaiC hexamers.

According to this model, hypophosphorylated $\mathrm{KaiC}$ associates with KaiA, which facilitates its phosphorylation; KaiB then binds hyperphosphorylated KaiC inducing a conformational change towards a new state in which KaiC autodephosphorylates and returns to the original conformation.

Because previous work indicated that KaiC hexamers undergo monomer exchange, the authors labelled two groups of KaiC hexamers with different fluorophores and used fluorescence resonance energy transfer to

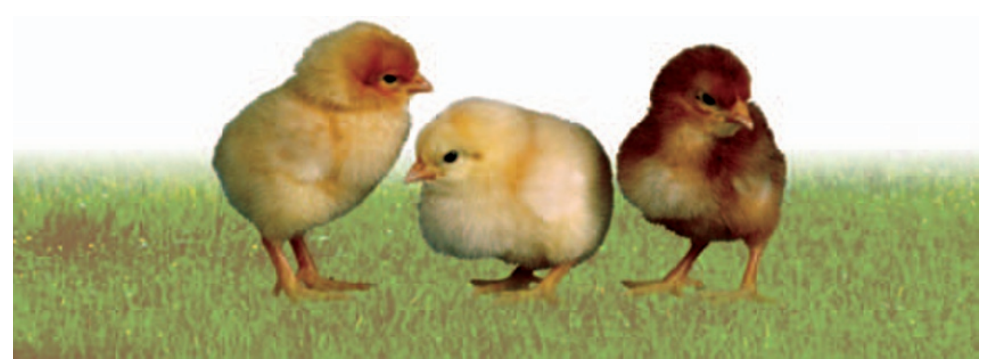

test this possibility. Indeed, monomer exchange occurred between KaiC hexamers, preferentially during the dephosphorylation phases of the reaction, and this was not inhibited by either KaiA or KaiB. This conformation-dependent monomer exchange was sufficient to generate sustained, high-amplitude oscillations in KaiC phosphorylation without the need for feedback loops.

Finally, using perturbation analyses, the authors showed that the in vitro KaiABC oscillator could also be reset in response to such stimuli as a temperature pulse.

Although it is still unclear how the KaiABC complex regulates the circadian output rhythms in vivo, this study provides an unprecedented insight into the molecular details of a circadian clockwork. The in vitro KaiABC interplay is also a nice example of an entirely posttranslational clock mechanism that well models the in vivo functioning of the cyanobacterial clock. For example, in conditions of constant darkness when transcription and translation are inhibited, the cyanobacterial clock relies only on post-translational mechanisms to mantain the circadian rhythm.

Francesca Pentimalli

ORIGINAL RESEARCH PAPER Mori, T. et al.

Elucidating the ticking of an in vitro circadian clockwork. PLoS Biol. 5, e93 (2007) FURTHER READING Bell-Pedersen D. et al. Circadian rhythms from multiple oscillators: lessons from diverse organisms. Nature Rev. Genet. 6, 544-556 (2005) 